\title{
Cuidado Paliativo e Diretrizes Curriculares: Inclusão Necessária
}

\section{Palliative Care and Curriculum Guidelines: Necessary Inclusion}

\author{
Paula Pereira Pineli, ${ }^{\text {III }}$ \\ Sara Krasilcic ${ }^{I I}$ \\ Fábio Akira Suzuki \\ Maria Goretti Sales Maciel ${ }^{\text {II }}$
}

\author{
PALAVRAS-CHAVE \\ - Cuidados Paliativos; \\ - Educação de Graduação em \\ Medicina; \\ - Currículo; \\ - Educação Médica.
}

Recebido em: 28/05/2015

Aprovado em: 11/04/2016

\begin{abstract}
RESUMO
Cuidado Paliativo (CP) é uma abordagem multiprofissional que visa à qualidade de vida de pacientes com doenças ameaçadoras de vida e de seus familiares. Com o avanço das doenças crônico-degenerativas, estima-se que mais de 20 milhões de pacientes no mundo têm necessidade deste perfil de cuidado. No que tange à formação médica direcionada a essa demanda crescente, há escassa menção nas Diretrizes Curriculares Nacionais do Curso de Graduação em Medicina. Contudo, ao se avaliar o texto oficial, observam-se diversas congruências das orientações gerais com os princípios do $C P$, como: formação humanística, priorização de pacientes em vulnerabilidade, valorização da dignidade humana, bioética, respeito à autonomia do paciente, abordagem centrada na pessoa, trabalho em equipe, abordagem familiar, comunicação, condutas baseadas em evidência. O estudo destes pontos em comum evidencia o CP como potencial espaço formador para aquisição de habilidades e competências requeridas pelas orientações oficiais. Neste sentido, a inserção na graduação ampliará a formação profissional de futuros médicos, podendo contribuir ainda com a garantia de uma assistência de qualidade a pacientes com doenças graves, em especial na fase final de vida.
\end{abstract}

\begin{abstract}
Palliative Care (PC) is a multidisciplinary team approach that deals with patients and their families under conditions of life-threatening illness aimed at improving their quality of life. With the increase of chronic-degenerative diseases, it is estimated that 20 million patients in the world require this type of care. With regard to medical training geared toward this demand there is scarce mention of $P C$ in the National Curriculum Guidelines for Undergraduate Medical Courses. However, when the official text is reviewed, several parallels can be drawn between the general guidelines and the principles of $P C$, such as humanistic training, prioritization of vulnerable patients, valuing human dignity, bioethics, respect for the patient's autonomy, person-centered approach, team work, family approach, communication and evidence-based practices. The study of these common points shows PC to be a potential educational strategy for the acquisition of required skills and competencies according to the official guidelines. In this regard, the inclusion of PC in undergraduate medical courses will broaden the professional training of future physicians, as well as helping guarantee quality care for seriously ill patients, especially those in the final stages of their lives.
\end{abstract}




\section{CUIDADO PALIATIVO: CONCEITO E DEMANDA} ATUAL

O Cuidado Paliativo (CP) teve início com o Movimento Hospice Moderno, em 1967, com a fundação do Saint Christopher's Hospice por Dame Cicely Saunders. Tal marco significou um olhar diferenciado sobre o paciente com doença avançada, sem perspectiva curativa, visando a um controle impecável dos sintomas, em especial da dor ${ }^{1}$.

Com o passar do tempo, sua definição foi sendo organizada, e até recentemente foi embasada pelo conceito de 2002 da Organização Mundial de Saúde:

O Cuidado Paliativo é uma abordagem que visa a melhorar a qualidade de vida dos pacientes e suas famílias que enfrentam alguma doença com risco de vida, através da prevenção e alívio do sofrimento, por meio da identificação precoce, avaliação impecável e tratamento da dor e de outros problemas físicos, psicossociais e espirituais ${ }^{2}$.

A Worldwide Pallitive Care Alliance (WPCA), em 2014, ampliou o conceito e definiu que o Cuidado Paliativo é necessário tanto para condições que limitam a vida quanto para doenças crônicas; e que não há um tempo de vida estimado ou um prognóstico que determinem a indicação de Cuidado Paliativo, mas, sim, a necessidade do paciente.

Sugere também que a capacitação dos profissionais que atuam na área deve ser dividida em três níveis de complexidade: (i) conceitos gerais na formação de todos profissionais da área da saúde; (ii) conhecimentos básicos para atuação de médicos generalistas envolvidos na Atenção Primária à Saúde; (iii) formação de especialistas para assistência de casos de alta complexidade ${ }^{3}$.

Atualmente, estima-se que $66 \%$ das pessoas vão morrer por doenças oncológicas e crônico-degenerativas, passando por longos períodos de sofrimentos passíveis de alívio. Portanto, não há como negar a premente necessidade deste cuidado. Levando-se em conta apenas a fase final de vida, estima-se que hoje 20 milhões de pessoas no mundo necessitam de cuidados paliativos ${ }^{3}$.

Considerando-se a vasta disponibilidade de tratamentos modificadores do curso das doenças e dos recursos avançados, cabe aos profissionais identificar o momento da doença em que os indivíduos se encontrem para tratá-los de forma proporcional ao prognóstico.

Segundo a Carta de Praga, a não disponibilização do Cuidado Paliativo para aqueles que estão em sofrimento por doença ameaçadora de vida é considerada hoje um tratamento "cruel, desumano ou degradante". Há um direcionamento para se cessar a prática da distanásia. $\mathrm{O}$ documento pontua ainda a necessidade de alterar a formação dos diferentes profissionais de saúde a fim de que se capacitem em CP para cuidar dos pacientes com esta demanda, independentemente do local ou nível de atenção ocupado por esses profissionais ${ }^{4}$.

De acordo com esta mudança de paradigma, o Conselho Federal de Medicina, em 2009, atualiza seu Código de Ética Médica e descreve objetivamente em seu artigo 41:

Nos casos de doença incurável ou terminal deve o médico
oferecer todos os cuidados paliativos disponíveis sem empre-
ender ações diagnósticas ou terapêuticas inúteis ou obstina-
das, levando sempre em consideração a vontade expressa do
paciente ou, na sua impossibilidade, a de seu representante
legal ${ }^{5}$ (p.51).

Ressalta-se a generalização da incumbência deste dever para todos os formados em Medicina, e não apenas a uma classe de especialistas.

Assim, diante de uma mudança no perfil de adoecimento, das demandas emergentes dos pacientes e das orientações de conduta estabelecidas por órgãos nacionais e internacionais, é inequívoca a importância da formação profissional para atender a este hiato ${ }^{6,7}$. Portanto, a atuação médica neste contexto, em qualquer espaço público ou privado, de maior ou menor complexidade, se deparará constantemente com a exigência do exercício de habilidades e competências em Cuidado Paliativo.

O presente trabalho se dispõe a avaliar as orientações oficiais para a formação médica de nosso país presentes nas Diretrizes Curriculares de 2014, com o intuito de avaliar as congruências e as lacunas condizentes com as necessidades dessa nova área de atuação na Medicina.

\section{DCN E O CUIDADO PALIATIVO}

Ao se avaliarem as Diretrizes Nacionais Curriculares (DCN) do curso de Medicina de 2001 e de 2014, observa-se uma importante escassez a respeito da necessidade de aprendizado do cuidado com doenças que colocam em risco a vida, em especial na sua fase final. As citações sobre a morte e o processo de terminalidade de vida, quando ocorrem, são breves e superficiais.

Nas DCN de 2001, apenas dois trechos contemplam tais menções. Um deles encontra-se nos itens sobre os conteúdos fundamentais para graduação médica: "promoção da saúde e compreensão dos processos fisiológicos dos seres humanos - gestação, nascimento, crescimento e desenvolvimento, envelhecimento e o processo de morte, atividades físicas, desportivas e as relacionadas ao meio social e ambiental" ${ }^{\prime \prime}(p .4)$. 
O outro se apresenta no artigo $5^{\circ}$, sobre as premissas de competências e habilidades para formação no ensino superior, mencionando, entre outros aspectos, o "acompanhamento do processo de morte" como parte integrante da atuação profissional do futuro médico" ${ }^{\prime \prime}(\mathrm{p} .3)$.

Nesta mesma linha, as DCN de 2014 também não se detêm na temática, reduzindo a referência somente ao seu artigo 23 , sobre os conteúdos fundamentais. Neste, o termo processo de morte (mais adequado) é simplificado apenas para o evento "morte": "promoção da saúde e compreensão dos processos fisiológicos dos seres humanos (gestação, nascimento, crescimento e desenvolvimento, envelhecimento e morte), bem como das atividades físicas, desportivas e das relacionadas ao meio social e ambiental" ${ }^{\prime \prime}$ (p.10).

O reflexo cultural de abstenção de tratar do tema que envolve o processo de morrer é claramente visualizado nestas propostas oficiais de formação médica. Mesmo sendo o lidar e o cuidar de pacientes na fase final de vida um evento recorrente na prática de qualquer médico, pouco se fala e se discute sobre o assunto, principalmente no processo educacional da graduação $7,10,11$.

Apesar deste contexto, é possível observar que as prerrogativas de conhecimentos, habilidades e competências exigidas no exercício da profissão vão ao encontro da formação ampla, questionadora e ética que se supõe no CP.

A seguir, serão citados diversos trechos das DCN de $2014^{9}$ nos quais há congruência com os princípios dos Cuidados $\mathrm{Pa}$ liativos, com posterior discussão.

(1) Capítulo I, artigo $3^{\circ}$, sobre as características requeridas para o médico em formação:

geral, humanista, crítica, reflexiva e ética, ...e compromisso com a defesa da cidadania, da dignidade humana... (p.1)

(2) Item I, artigo $5^{\circ}$ da Seção I, sobre a responsabilidade da concretização da cidadania inerente ao Sistema Único de Saúde, ressaltando a priorização dos indivíduos em vulnerabilidade:

acesso universal e equidade como direito à cidadania, sem privilégios nem preconceitos de qualquer espécie, tratando as desigualdades com equidade e atendendo as necessidades pessoais específicas, segundo as prioridades definidas pela vulnerabilidade e pelo risco à saúde e à vida, observado o que determina o Sistema Único de Saúde (SUS) (p.2)

(3) Artigo 5o da Seção I sobre o entendimento da assistência alicerçado não apenas em sinais e sintomas físicos, mas também em todas as diversas dimensões do indivíduo:
Na Atenção à Saúde, o graduando será formado para considerar sempre as dimensões da diversidade biológica, subjetiva, étnico-racial, de gênero, orientação sexual, socioeconômica, política, ambiental, cultural, ética e demais aspectos que compõem o espectro da diversidade humana que singularizam cada pessoa ou cada grupo social (p.1 e 2)

(4) Item II do artigo $5^{\circ}$ da Seção I sobre a valorização da autonomia do indivíduo, como protagonista na tomada de decisões em sua vida, inclusive no processo de adoecer:

estimulando o autocuidado e a autonomia das pessoas, famílias, grupos e comunidades e reconhecendo os usuários como protagonistas ativos de sua própria saúde (p.2)

(5) Item VII do artigo $5^{\circ}$ da Seção I e item IV do artigo 6으 da Seção II sobre a importância da habilidade em comunicação:

comunicação, por meio de linguagem verbal e não verbal, com usuários, familiares, comunidades e membros das equipes profissionais, com empatia, sensibilidade e interesse, preservando a confidencialidade, a compreensão, a autonomia e a segurança da pessoa sob cuidado (p.2)

Comunicação, incorporando, sempre que possível, as novas tecnologias da informação e comunicação (TICs), para interação a distância e acesso a bases remotas de dados (p.3)

(6) Item II do artigo 5ㅜ, item IX do artigo 5ㅜ, Seção I, item VI do artigo $6^{\circ}$ da Seção II e item III do artigo $7^{\circ}$ da Seção III sobre a capacitação para o trabalho em equipe, visando à construção em conjunto de planos terapêuticos:

integralidade e humanização do cuidado por meio de prática médica contínua e integrada com as demais ações e instâncias de saúde, de modo a construir projetos terapêuticos compartilhados (p.2)

cuidado centrado na pessoa sob cuidado, na família e na comunidade, no qual prevaleça o trabalho interprofissional, em equipe, com o desenvolvimento de relação horizontal, compartilhada, respeitando-se as necessidades e desejos da pessoa sob cuidado, família e comunidade, a compreensão destes sobre o adoecer, a identificação de objetivos e responsabilidades comuns entre profissionais de saúde e usuários no cuidado (p.2)

Trabalho em Equipe, de modo a desenvolver parcerias e constituição de redes, estimulando e ampliando a aproximação 
entre instituições, serviços e outros setores envolvidos na atenção integral e promoção da saúde (p.3)

aprender interprofissionalmente, com base na reflexão sobre a própria prática e pela troca de saberes com profissionais da área da saúde e outras áreas do conhecimento, para a orientação da identificação e discussão dos problemas, estimulando o aprimoramento da colaboração e da qualidade da atenção à saúde (p.3)

(7) Item III do artigo 5ำ da Seção I, item III do artigo $6^{\circ}$ da Seção II e parte C do item I do artigo 12 sobre o cuidar impecável pautado em conhecimento técnico, com evidências científicas, que mudam/agregam novos olhares, cuidados e medidas terapêuticas, visando ao zelo proporcional, em especial nas situações de vulnerabilidade (citados na ordem acima):

qualidade na atenção à saúde, pautando seu pensamento crítico, que conduz o seu fazer, nas melhores evidências científicas, na escuta ativa e singular de cada pessoa, família, grupos e comunidades e nas políticas públicas, programas, ações estratégicas e diretrizes vigentes (p.2)

Tomada de Decisões, com base na análise crítica e contextualizada das evidências científicas, da escuta ativa das pessoas, famílias, grupos e comunidades, das políticas públicas sociais e de saúde, de modo a racionalizar e otimizar a aplicação de conhecimentos, metodologias, procedimentos, instalações, equipamentos, insumos e medicamentos, de modo a produzir melhorias no acesso e na qualidade integral à saúde da população e no desenvolvimento científico, tecnológico e inovação que retroalimentam as decisões (p.3)

orientação do atendimento às necessidades de saúde, sendo capaz de combinar o conhecimento clínico e as evidências científicas, com o entendimento sobre a doença na perspectiva da singularidade de cada pessoa (p.5)

\section{DCN E CUIDADO PALIATIVO: CONGRUÊNCIAS E LACUNAS}

É possível identificar congruências em diferentes aspectos nas três Seções das DCN de 2014, Atenção à Saúde, Gestão em Saúde, Educação em Saúde, nos trechos supracitados, como podemos observar nos Princípios dos mesmos, apresentados a seguir:

(1) Promover o alívio da dor e de outros sintomas desagradáveis;
(2) Afirmar a vida e considerar a morte como um processo normal da vida;

(3) Não acelerar nem adiar a morte;

(4) Integrar os aspectos psicológicos e espirituais no cuidado ao paciente;

(5) Oferecer um sistema de suporte que possibilite ao paciente viver tão ativamente quanto possível até o momento da sua morte;

(6) Oferecer um sistema de suporte para auxiliar os familiares durante a doença do paciente e ao enfrentar o luto;

(7) Abordagem multiprofissional para focar as necessidades dos pacientes e seus familiares, incluindo acompanhamento no luto;

(8) Melhorar a qualidade de vida e influenciar positivamente o curso da doença;

(9) Iniciar o mais precocemente possível, juntamente com outras medidas de prolongamento da vida, como a quimioterapia e a radioterapia, e incluir todas as investigações necessárias para melhor compreender e controlar situações clínicas estressantes $^{12}$.

Assim, ao se propiciar que discentes, em seu processo de formação, tenham vivências com pacientes com doenças que ameacem a vida desde o diagnóstico até o final da vida, pautados na observância destes princípios, viabilizam-se experiências coerentes com as atuais diretrizes.

O profissional de saúde (graduado ou em formação), ao se deparar com o cuidado de pacientes, em especial na última fase da vida, é convocado, continuamente, a ter uma postura humanística ${ }^{10}$. A individualidade e as peculiaridades que marcam esta experiência única para cada pessoa exigem daquele responsável por seu cuidado uma contínua reflexão e adaptação das medidas terapêuticas. $\mathrm{O}$ foco no alívio dos sintomas que propicie qualidade de vida deixa as medidas protocolares em segundo plano, colocando em evidência a demanda daquele a ser cuidado. Um exemplo do benefício deste campo de atuação é o relato de experiências práticas com discentes em contato direto com o processo de morrer, demonstrando evolução positiva nas atitudes dos mesmos frente a tal situação ${ }^{13}$.

Diante da fragilidade progressiva que marca o processo em si, evidencia-se a garantia de um direito de cidadania dos mais básicos: a manutenção da dignidade humana. Assim, a extrema vulnerabilidade deste indivíduo, muitas vezes associada a sofrimento intenso, dá um caráter urgente a sua necessidade de atendimento, mesmo que sem risco iminente de vida, sendo este um importante critério de priorização ${ }^{11}$.

Neste contexto, é possível observar ainda que o exercício da ética encontra questões mais delicadas, recorrentes e confli- 
tivas. Decisões e deliberações são exigidas de forma cotidiana ${ }^{7}$. A fragilidade humana, somada à vulnerabilidade da dignidade humana, continuamente requer uma revisão de prescrições e de condutas, priorizando-se a vontade do indivíduo que é o foco do cuidado. A anteposição da escolha desse indivíduo exige o redimensionamento do valor de medidas prolongadoras de vida, da opinião de familiares, bem como dos valores morais de quem cuida. Propicia-se, assim, um debate contínuo destas questões e da bioética em si, temas cada vez mais fundamentados como importantes desde a graduação ${ }^{14,15}$.

Identifica-se então um espaço formador que permite o exercício das qualidades exigidas para um médico humanista, crítico, reflexivo e ético, pautado em aspectos primordiais da cidadania, inclusive no SUS.

Sobre o foco do tratamento dos pacientes, visa-se ao alívio dos sintomas não apenas físicos, mas também psicológicos, espirituais e de outras dimensões que ocasionem sofrimento ${ }^{10,11}$. Assim, a valorização da biografia individual integrada ao contexto familiar e comunitário do indivíduo, entendendo os aspectos socioculturais e socioeconômicos que o influenciam, permite conhecer os determinantes de sua qualidade de vida. Por meio destas informações é possível cuidar da pessoa em si, e não somente do processo patológico que a acomete. Educar o aluno nesta perspectiva facilita o rompimento com o modelo centrado na doença para o centrado na pessoa ${ }^{11,16,17}$.

A fim de garantir o atendimento desta globalidade e integralidade, o exercício dos Cuidados Paliativos é por essência multiprofissional ${ }^{15,16}$, como também observado em seus princípios. Somente com um atendimento composto por profissionais de diferentes profissões é possível cuidar das diferentes necessidades do indivíduo e também de sua família. Logo, inserir um profissional em formação neste contexto lhe propicia a experiência de trabalho em equipe. A inserção precoce no processo formativo favorece ainda um aprendizado inicial já com esta característica, fugindo de vícios de individualismo, sendo ideal se atrelado a um contexto de prática clínica ${ }^{15}$.

Além disso, toda a atuação junto ao paciente deve respeitar a vontade dele, tendo a mesma como norteadora na tomada de decisões. Qualquer profissional envolvido em atendimentos de diferentes tipos deve ter profundo conhecimento da pessoa que está assistindo e de suas escolhas. A autonomia do paciente é pedra angular que direciona as medidas que visam à qualidade de vida ${ }^{16}$. Sendo assim, é inequívoca a participação ativa do indivíduo em todo o processo propedêutico e terapêutico, e, quando pertinente, a inclusão também dos familiares e cuidadores ${ }^{7,11}$. De forma concreta, tal princípio se estabelece mediante a realização e o respeito das Diretivas Antecipadas de Vontade, com documentação em prontuário do desejo do indivíduo em relação às possíveis condutas a que possa ser submetido, respaldadas pelo Conselho Federal de Medicina em sua Resolução 1.995/2012 ${ }^{18}$.

Outra importante habilidade que perpassa todas as citadas é a de comunicação, que atualmente tem sido identificada como o nó crítico dos conflitos na relação médico-paciente ${ }^{10}$. $\mathrm{Na}$ área da saúde, durante todo o processo de cuidar de alguém com doença grave ou potencialmente grave, o profissional se depara invariavelmente com a necessidade de informar más notícias. Assim, a habilidade de comunicação trata-se de outro pilar norteador ${ }^{10,15,16,19}$. Neste sentido, não há como pensar a formação médica sem a aquisição do domínio deste requisito.

No que tange à fundamentação teórica para a prática clínica, a exigência de qualidade na assistência prestada é um dos pilares nos Cuidados Paliativos. Isto se justifica porque, frente a um indivíduo frágil, a escolha da melhor conduta garante a integridade deste. Há uma preocupação ininterrupta de ponderar todos os pormenores em todas as condutas. A avaliação do real benefício integra-se à ponderação de riscos, e a indicação de medidas ditas "protocolares" deve ser analisada para cada pessoa em seu processo individual de adoecer e de morrer. Os recorrentes norteadores em qualquer prática nesta área de atuação - "atenção aos detalhes" e "reavaliação contínua" - evidenciam este perfil de cuidado, tendo o paciente como centro do cuidado, e não a conduta em si ${ }^{12}$.

Diante destes diferentes aspectos abordados, é possível demonstrar o campo de atuação dos CP como um espaço de formação que atende às orientações oficiais em diversos itens. Além disso, trata-se de um espaço único de aprendizagem de manejo de doenças que podem limitar a sobrevida, em especial nas fases de pouco ou nenhum tratamento que lhes mude o curso. Apesar da identificação da grande importância destas vivências em diferentes países ${ }^{14,15,16,21}$, estudos realizados no Brasil demonstram a escassez de aproveitamento deste perfil de vivência nos cursos de graduação ${ }^{7,20}$.

\section{CONCLUSÕES E PERSPECTIVAS}

Embora não haja uma orientação curricular de inserção da temática dos Cuidados Paliativos nos cursos de Medicina do nosso país, é possível traçar pontos em comum entre as atuais DCN e as prerrogativas deste campo de atuação. A avaliação realizada demonstrou que a prática no contexto dos $\mathrm{CP}$ se mostra uma importante área de aprendizado na formação médica no que tange tanto aos cuidados de fim de vida, quanto à formação global em diferentes requisitos. Apesar do número limitado de experiências neste sentido no Brasil, o vislumbre da construção desta possibilidade de trajetória abre espaço para novas iniciativas e propostas no meio acadêmico. 
Assim, é possível ter expectativas não apenas de ampliar o conhecimento médico que contemple este contexto, mas, de forma mais ampla, garantir a qualidade da assistência prestada aos pacientes com doenças ameaçadoras da vida, principalmente em sua fase final.

Entender a importância da incorporação da temática no processo formativo propiciará novos debates que abrirão espaço para o atendimento de uma demanda epidemiológica em crescimento significativo.

\section{REFERÊNCIAS}

1. Twycross R. Medicina Paliativa: Filosofia e Considerações Éticas. Acta Bioethica. 2000; 4(1):1-24.

2. Organização Mundial de Saúde [homepage]. Acesso em 02 ago. 2014. Disponível em: http://www.who.int/cancer/ palliative/definition/en/

3. Organização Mundial de Saúde. Global Atlas of Palliative Care at the end-of-life. Acesso em 02 dez. 2014. Disponível em: http://www.who.int/nmh/Global_Atlas_of_Palliative_Care.pdf

4. European Association for Palliative Care [homepage]. Carta de Praga. Acesso em 02 dez. 2014. Disponível em: http:/ / www.eapcnet.eu/LinkClick.aspx?fileticket=6AmB AJNDb8A\%3D\&tabid=1871

5. Conselho Federal de Medicina. Código de ética médica: Resolução CFM no 1931, de 17 de setembro de 2009 (versão de bolso). Brasília: Conselho Federal de Medicina, 2010. 98 .

6. Gamondi C, Larkin P, Payne S. Competências Centrais em Cuidados Paliativos: Um Guia Orientador da EAPC sobre Educação em Cuidados Paliativos - parte 1. European Journal of Palliative Care, 2013; 20(2):86-91.

7. Oliveira JR, Ferreira AC, Rezende NA. Ensino de Bioética e Cuidados Paliativos nas Escolas Médicas do Brasil. Revista Brasileira de Educação Médica 2013; 37(2):285-290.

8. Brasil. Ministério da Educação. Conselho Nacional de Educação. Câmara de Educação Superior. Resolução CNE/ CES nº 4, de 7 de novembro de 2001. Institui diretrizes curriculares nacionais do curso de graduação em Medicina. Diário Oficial da União. Brasília, 9 nov. 2001; Seção 1, p.38.

9. Brasil. Ministério da Educação. Conselho Nacional de Educação. Câmara de Educação Superior. Resolução CNE/CES nㅜㄹ, de 20 de junho de 2014. Institui diretrizes curriculares nacionais do curso de graduação em Medicina e dá outras providências. Diário Oficial da União. Brasília, 23 jun. 2001; Seção 1, p.8-11.

10. Azeredo NSG, Rocha CF, Carvalho PRA. O Enfrentamento da Morte e do Morrer na Formação de Acadêmicos de
Medicina. Revista Brasileira de Educação Médica 2011; 35(1):37-43.

11. Figueiredo MGMCA, Stano RCMT. O Estudo da Morte e dos Cuidados Paliativos: uma Experiência Didática no Currículo de Medicina. Revista Brasileira de Educação Médica 2013; 37(2):298-307.

12. Carvalho RT, Parsons HA. Manual de Cuidados Paliativos ANCP. 2.ed. Porto Alegre: Sulina; 2012.

13. Anderson WG, Williams JE, Bost JE, Barnard D. Exposure to Death is Associated with Positive

14. Attitudes and Higher Knowledge About End-of-Life Care in Graduating Medical Students. Journal of Palliative Medicine 2008; 11(9):1227-1233.

15. Gruber PC, Gomersall CD, Joynt GM, Lee A, Tang PYG, Young AS, Florrie Yu NY,Ting Yu O. Changes in Medical Students' Attitudes Towards End-of-Life Decisions Across Different Years of Medical Training. Journal of General Internal Medicine 2008; 23(10):1608-1614.

16. Hillier R, Wee B. From cradle to grave: palliative medicine education in the UK. Journal of the Royal Society of Medicine 2001; 94:468-471.

17. Grant M, Elk R, Ferrell B, Morrison RS, Gunten CF. Current Status of Palliative Care, Education, and Research. CA: A Cancer Journalfor Clinicans 2009; 59(5):327-335.

18. Block SD, Bernier GM, Crawley LM, Faber S, Kuhl D, Nelson W, O’Donnell J, Sandy L, Ury W. Incorporating Palliative Care into Primary Care Education. Journal of General Internal Medicine 1998; 13:768-773.

19. Conselho Federal de Medicina. Resolução CFM nº 1.995/2012. Dispõe sobre as diretivas antecipadas de vontade dos pacientes. Diário Oficial da União. Brasília, 31 ago. 2012; Seção 1, p.269-270

20. Sullivan AM, Lakoma MD, Block DS. The status of medical education in end-of-life care. A National report. Journal of General Internal Medicine 2003; 18:685-695.

21. Toledo AP, Priolli DG. Cuidados no Fim da Vida: O Ensino Médico no Brasil. Revista Brasileira de Educação Médica 2012; 36(1):109-117.

22. Ilse B, Hildebrandt J, Posselt J, Laske A, Dietz I, Borasio GD, Kopf A, Nauck F, Elsner F, Wedding U, Alt-Epping B. Palliative Care teaching in Germany - concepts and future developments. GMS Zeitschrift für Medizinische Ausbildung 2012; 29(3):7-12.

\section{CONTRIBUIÇÃO DOS AUTORES}

Paula Pereira Pineli foi responsável principal pela revisão da temática e elaboração do texto em parceria com Sara Krasilcic. Maria Goretti Sales Maciel e Fábio Akira Suzuki fizeram a re- 
visão final do texto elaborado, contribuindo com orientações que direcionaram reestruturação de diferentes trechos.

\section{CONFLITO DE INTERESSES}

Não há.

\section{ENDEREÇO PARA CORRESPONDÊNCIA}

Paula Pereira Pineli

Avenida Ibirapuera, 981 - sala 348

Indianópolis - São Paulo

CEP 04028-000 - SP

E-mail: paula.pineli@ufv.br 